Pesq. Vet. Bras. 30(12):1017-1020, dezembro 2010

\title{
Intoxicação espontânea por larvas de Perreyia flavipes (Pergidae) em suínos no Estado de Santa Catarina ${ }^{1}$
}

\author{
Fernanda Jonck², Renata Assis Casagrande ${ }^{2}$, Diego Lacir Froehlich², Djalmo \\ Pereira Ribeiro Junior ${ }^{3}$ e Aldo Gava ${ }^{2 *}$
}

\begin{abstract}
Jonck F., Casagrande R.A., Froehlich D.L., Ribeiro Jr D.P. \& Gava A. 2010. [Spontaneous poisoning by larvae of Perreyia flavipes (Pergidae) in pigs in the State of Santa Catarina.] Intoxicação espontânea por larvas de Perreyia flavipes (Pergidae) em suínos no Estado de Santa Catarina. Pesquisa Veterinária Brasileira 30(12):1017-1020. Departamento de Medicina Veterinária, Centro de Ciências Agroveterinárias, Universidade do Estado de Santa Catarina, Lages, SC 88520-000, Brazil. E-mail: a2ag@cav.udesc.br

The study reports an outbreak of spontaneous poisoning by Perreyia flavipes in pigs. The outbreak occurred at the end of May 2009, in the municipality of Urubici, plateau of Santa Catarina, Brazil. The farm had about 50 pigs reared extensively and 10 animals got sick. The clinical signs were anorexia, apathy, constant movement of the head and hitting the ears, difficulty to walk and stagger, gnashing of teeth and agitation that increased with noise and movement nearby. At necropsy, pronounced hepatic lobular pattern and $P$. flavipes larvae mixed with the stomach content were observed. Microscopically, hepatocellular centrilobular to diffuse coagulation necrosis with severe congestion and hemorrhage was observed, with vacuolar degeneration in one or two layers of hepatocytes in the portal zones. Clinical signs, epidemiology and lesions in the pigs were characteristic of toxic hepatitis by larvae of $P$. flavipes.
\end{abstract}

INDEX TERMS: Perreyia flavipes, Pergidae, sawfly, liver necrosis, pigs.

RESUMO.- Descreve-se um surto de intoxicação espontânea por Perreyia flavipes em suínos. O surto ocorreu no final de maio de 2009, na cidade de Urubici, planalto serrano do Estado de Santa Catarina, Brasil. A propriedade tinha aproximadamente 50 animais criados extensivamente e desses 10 animais adoeceram. Esses suínos apresentavam anorexia, apatia, movimento constante de cabeça e bater de orelhas, dificuldade de caminhar, cambaleio, ranger de dentes e a agitação aumentava mediante ruídos e movimentos próximos. Na necropsia as alterações observadas foram a marcada evidenciação do padrão lobular hepático e a presença de larvas de $P$. flavipes misturadas ao conteúdo estomacal. Microscopicamente observou-se

\footnotetext{
${ }^{1}$ Recebido em 7 de julho de 2010.

Aceito para publicação em 2 de agosto de 2010.

${ }^{2}$ Laboratório de Patologia Animal, Departamento de Clínica e Patologia, Centro de Ciências Agroveterinárias (CAV), Universidade do Estado de Santa Catarina (UDESC), Av. Luiz de Camões 2090, Bairro Conta Dinheiro, Lages, SC 88520-000, Brasil. *Autor para correspondência: a2ag@cav.udesc.br

${ }^{3}$ Médico Veterinário, Cooperativa de Produtores Rurais de Urubici, Av. Rodolfo Andermann 2580, Urubici, SC 88650-000, Brasil.
}

necrose de coagulação dos hepatócitos, com distribuição centrolobular a massiva que era acompanhada de congestão e hemorragia acentuada, restando uma ou duas camadas de hepatócitos com degeneração vacuolar na região portal. Os aspectos clínicos, epidemiológicos e as lesões caracterizaram hepatite tóxica por larvas de $P$. flavipes em suínos.

TERMOS DE INDEXAÇÃO: Perreyia flavipes, Pergidae, bicho mata-porco, necrose hepática, suínos.

\section{INTRODUÇÃO}

Perreyia flavipes (bicho mata-porco, bicho-da-chuva, "sawfly", lagarta preta, ruga) são insetos semelhantes às vespas, pertencentes à ordem Hymenoptera e subordem Symphuta. A ingestão da fase larval desses insetos tem sido reportada como causa de necrose hepática aguda em bovinos (Dadswell et al. 1984) e ovinos (McKenzie et al. 1985) na Austrália, em ovinos e caprinos na Dinamarca (Tramsborg et al. 1987) e em bovinos no Uruguai (Dutra et al. 1997). No Brasil, a intoxicação espontânea por larvas de $P$. flavipes foi relatada em bovinos (Dutra et al. 1997, 
Riet-Correa et al. 1998, Soares et al. 2008, Raymundo et al. 2009 ) e ovinos (Dutra et al. 1997, Riet-Correa et al. 1998, Raymundo et al. 2008).

Na espécie suína a intoxicação é descrita apenas experimentalmente (Riet-Correa et al. 1998, Soares et al. 2001). Embora existam casos sugestivos de intoxicação natural por larvas de $P$. flavipes, estes não foram descritos ou não tiveram diagnóstico confirmado (Costa Lima 1941, Camargo 1956). A intoxicação ocorre nos meses de maio a julho que corresponde ao período larval do inseto (Camargo 1956). Os animais ingerem a forma larval que é palatável (Soares 2001). São observadas colunas contendo inúmeras larvas que rastejam sobre a pastagem (Camargo 1956) principalmente nas primeiras horas da manhã, ou enquanto há umidade na pastagem (Soares et al. 2008).

Este trabalho tem o objetivo de descrever os aspectos clínicos e patológicos de um surto de intoxicação espontânea por larvas de Perreyia flavipes em suínos no Estado de Santa Catarina.

\section{MATERIAL E MÉTODOS}

Os dados epidemiológicos da intoxicação espontânea por Perreyia flavipes em suínos e sinais clínicos foram obtidos junto ao proprietário e ao veterinário responsável e em visita à propriedade onde ocorreu o surto no Estado de Santa Catarina. Para o estudo da patologia três suínos afetados foram necropsiados e fragmentos de encéfalo, pulmão, coração, fígado, baço, linfonodos, estômago, intestinos, rins e músculo esquelético foram coletados, fixados em formalina tamponada a $10 \%$, processados rotineiramente e corados pelo método de Hematoxilina e Eosina (HE) para leitura em microscopia óptica.

\section{RESULTADOS}

O surto da intoxicação espontânea por Perreyia flavipes ocorreu no final do mês de maio de 2009, em uma propriedade na cidade de Urubici, no Planalto Catarinense, onde eram criados extensivamente, aproximadamente cinquenta

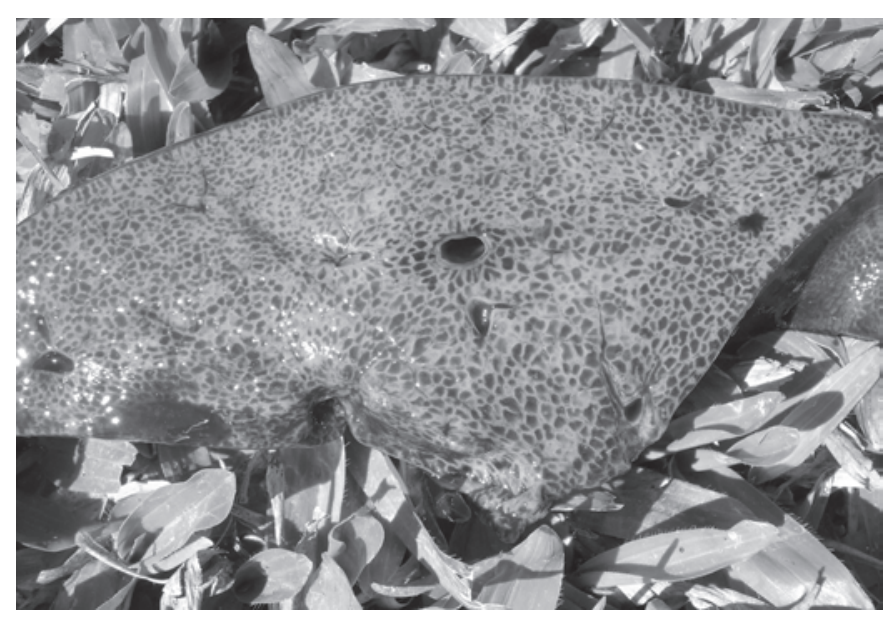

Fig.1. Superfície de corte do fígado com marcada evidenciação do padrão lobular, caracterizada por áreas vermelho-escuras delimitadas por linhas claras, na intoxicação espontânea por Perreyia flavipes em suíno. suínos da raça Landrace, jovens e adultos. Estes animais eram divididos em lotes e tinham acesso a pastagem existente entre os pomares de macieira durante o dia. À noite eram mantidos em baias e recebiam milho moído para alimentação. Segundo informações do proprietário a doença vinha sendo observada há vários anos nesta mesma época quando os suínos eram soltos no pomar de maçãs após a colheita.

Os sinais clínicos foram observados ao final do dia quando os animais eram recolhidos e consistiam de anorexia, apatia, movimento constante de cabeça e bater de orelhas. Apresentavam, ainda, dificuldade de caminhar e cambaleio, ranger de dentes e a agitação das orelhas e cabeça os quais aumentavam mediante ruídos e movimentos próximos. 0 período entre o início dos sinais clínicos e a morte dos animais foi de 12-24 horas. Adoeceram 10 suínos e desses, sete morreram e três se recuperaram. Na inspeção do pomar durante a visita a propriedade onde ocorreram as mortes não foram encontradas as larvas. No entanto, o proprietário informou que todos os anos nessa mesma época, as lagartas são observadas com frequência.

Foram necropsiados três suínos que morreram com os sinais clínicos da doença e que estavam em bom estado
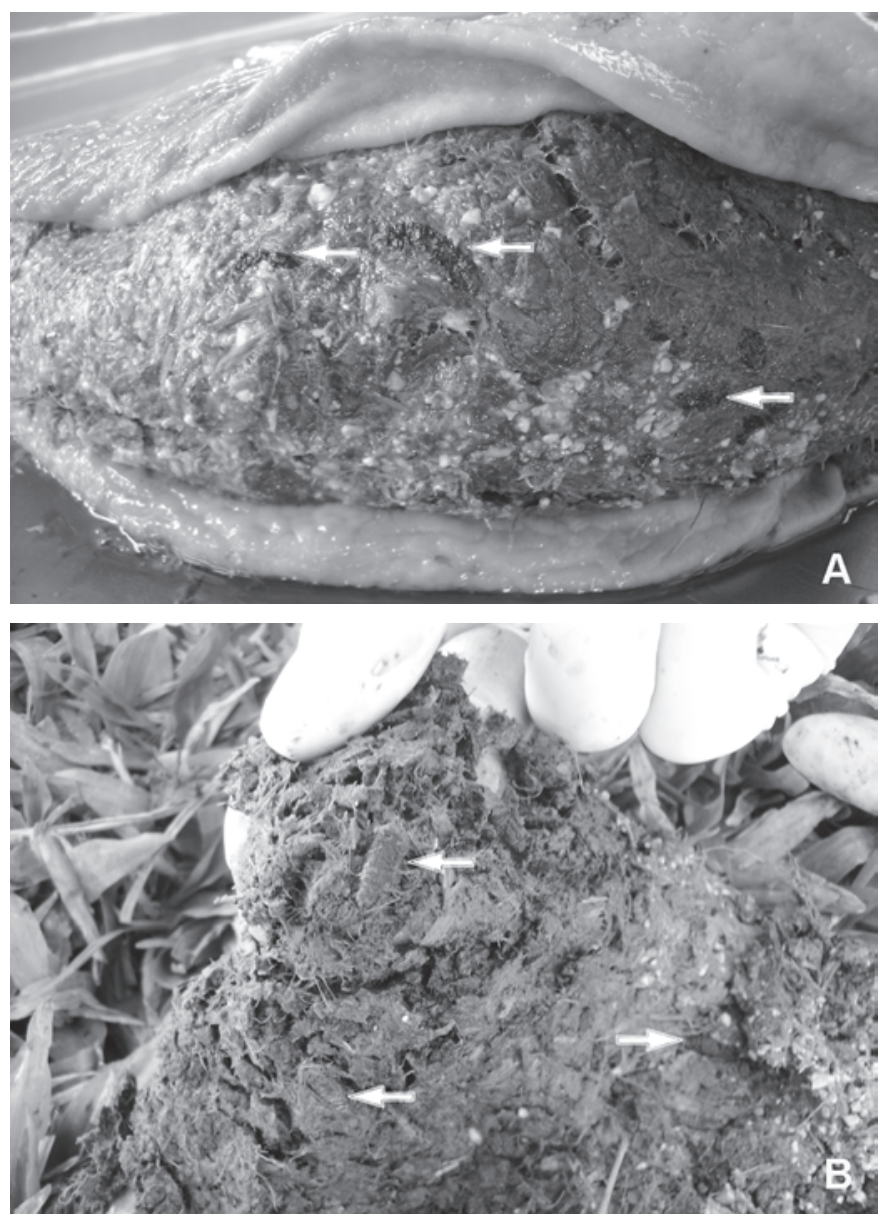

Fig.2. (A) Estômago contendo fragmentos e larvas de Perreyia flavipes. (B) Evidenciação das larvas no conteúdo estomacal, na intoxicação por Perreyia flavipes em suíno. 
nutricional. O fígado apresentava acentuação do padrão lobular evidente na superfície capsular e de corte que se caracterizava por áreas vermelho escuras, levemente deprimidas e delimitadas por linhas claras (Fig.1). No estômago havia inúmeros fragmentos de larvas e/ou larvas inteiras de $P$. flavipes misturadas ao conteúdo estomacal (Fig.2). Adicionalmente, havia fezes ressecadas no intestino grosso.

As lesões histológicas estavam restritas ao fígado e caracterizaram-se por necrose de coagulação dos hepatócitos, com distribuição centrolobular a massiva que era acompanhada de congestão e hemorragia acentuada. Estas áreas de necrose, quando centrolobulares, eram circundadas por uma ou duas camadas de hepatócitos com degeneração vacuolar (Fig.3).
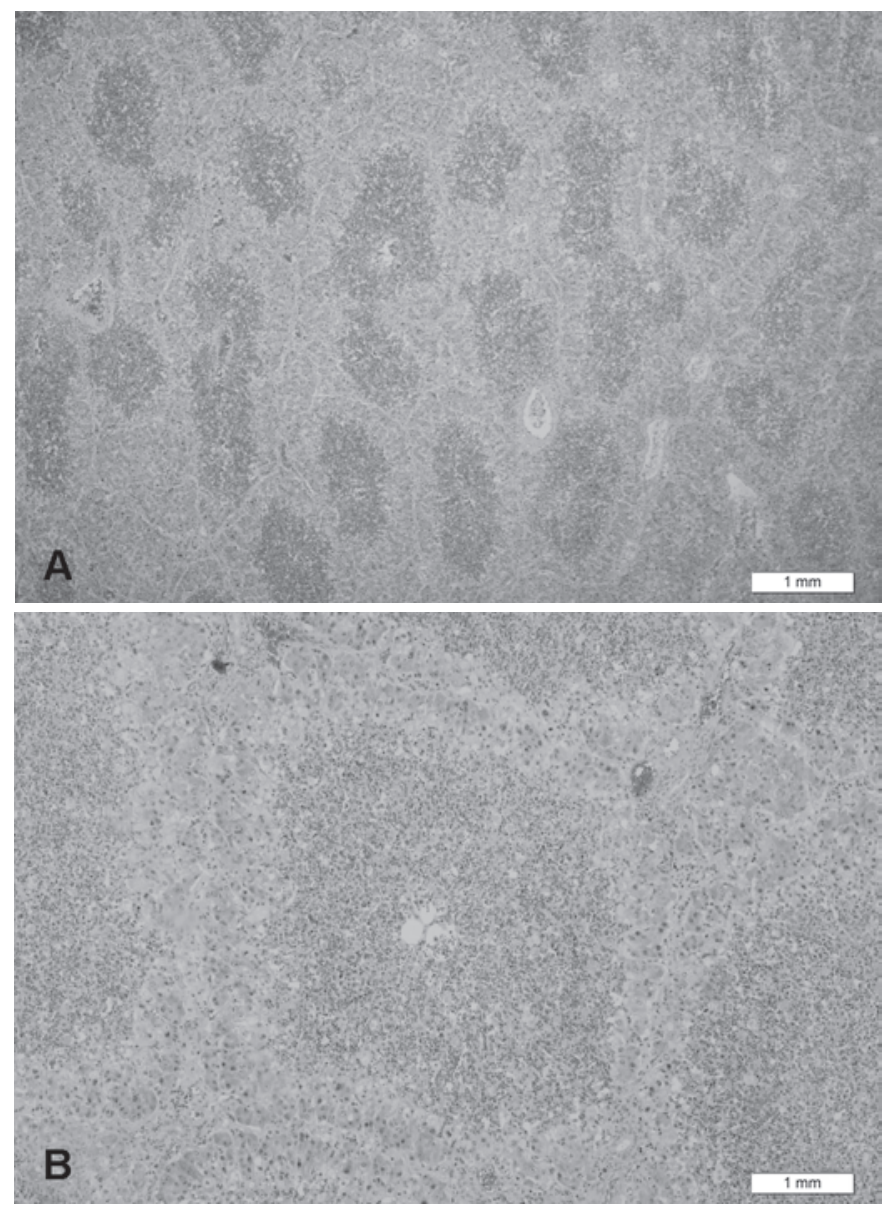

Fig.3. (A) Necrose de coagulação centrolobular no fígado acompanhada de congestão e hemorragia acentuadas, na intoxicação espontânea por larvas de Perreyia flavipes em suíno. HE, obj.4x. (B) Maior aumento da lesão. HE, obj.10x.

\section{DISCUSSÃO}

O diagnóstico de necrose hepática tóxica aguda associada ao consumo de larvas de $P$. flavipes baseou-se nos sinais clínicos, achados macroscópicos e microscópicos e pela visualização das larvas do inseto no conteúdo do estômago dos suínos mortos. Estes sinais clínicos, le- sões macroscópicas e microscópicas são semelhantes aos descritos na intoxicação por $P$. flavipes em bovinos (Dutra et al. 1997, Riet-Correa et al. 1998, Soares et al. 2008, Raymundo et al. 2009), ovinos (Dutra et al. 1997, Raymundo et al. 2008), caprinos (Tramsborg et al. 1987) e experimentalmente em suínos (Soares et al 2001).

O curso clínico observado neste surto foi de 12-24 horas. Em ruminantes é relatado que os animais intoxicados morrem entre 24-48 horas após o início dos sinais clínicos (Raymundo et al. 2008, Soares et al. 2008). Segundo Soares et al. (2001) na intoxicação experimental em suínos, utilizando $10 \mathrm{~g} / \mathrm{kg}$ de peso corporal (pc), de larvas frescas os sinais clínicos iniciaram 48 horas após a ingestão e a morte ocorreu 4 dias após. Neste mesmo estudo um suíno que recebeu $5 \mathrm{~g} / \mathrm{kg}$ de $\mathrm{PC}$ de larvas frescas em dose única, apresentou anorexia e depressão em 48 horas, no entanto, recuperou-se em 5 dias. No presente surto, observou-se que três animais adoeceram e recuperaram-se dias após. Isso certamente deve-se a quantidade de larvas ingeridas, bem como a fatores intrínsecos de cada animal.

Os distúrbios neuromusculares manifestados pelos suínos no presente trabalho foram observados, também, em bovinos, ovinos e experimentalmente em suínos intoxicados por essa lagarta (Dutra et al. 1997, Riet-Correa et al. 1998, Soares et al. 2001, Soares et al. 2008, Raymundo et al. 2008, Raymundo et al. 2009). Estes sinais caracterizam a encefalopatia hepática e podem ser conseqüência de hipoglicemia ou fatores detoxificantes do sistema hepático, levando ao acúmulo dos aminoácidos e amônia, ou de acetilcolina, ou liberação de produtos de degradação (Radostits et al. 2002). A encefalopatia hepática pode apresentar lesões como edema cerebral, necrose e tumefação de neurônios, e degeneração dos astrócitos, porém podem ocorrer casos sem a presença de lesões (Jones et al. 2000). Alterações microscópicas não foram observadas no sistema nervoso central dos animais do presente relato, o que pode ser explicado pela evolução clínica rápida e morte dos animais.

Outras alterações como icterícia discreta, fotossensibilização e hemorragias em serosas e mucosas do tubo digestivo e no coração são descritas na intoxicação espontânea e experimental por $P$. flavipes em bovinos e ovinos (Riet-Correa et al. 1998, Soares et al. 2008, Raymundo et al. 2008, Raymundo et al. 2009). Na intoxicação experimental em suínos é descrito leve icterícia e não há referências a hemorragias (Soares et al. 2001). Nenhuma dessas alterações foi observada no surto aqui descrito.

Além da necrose hepática são descritos necrose e depleção dos centros germinativos do baço e linfonodos (McKenzie et al. 1985, Dutra et al. 1997, Soares et al. 2001, 2008, Raymundo et al. 2008, 2009). Neste estudo, não foram encontradas lesões em órgãos linfóides.

O surto ocorreu no período de maio a julho que para o estado de Santa Catarina corresponde com o período larval do inseto. As larvas de $P$. flavipes não foram observadas na propriedade. Isso pode ter ocorrido, devido a que estes grupos de larvas movimentam-se constantemente e são 
observados principalmente nas primeiras horas do dia (Dutra et al. 1997, Riet-Correa et al. 1998, Soares et al. 2001). No entanto, na necropsia de todos os animais havia larvas misturadas ao conteúdo estomacal.

A intoxicação por larvas de $P$. flavipes em suínos não é freqüente, pois, essa espécie geralmente é criada em sistema intensivo. Porém, nas propriedades com criação extensiva ou, em sistema de criação "Plainer", usado em algumas regiões do estado de Santa Catarina, os suínos podem ter acesso às larvas. $O$ hábito de fuçar e remexer dos suínos facilita que estes encontrem as larvas e as consumam, visto que são palatáveis. Este fato foi evidenciado durante a intoxicação experimental em suínos, após ingerirem as larvas misturadas à ração passaram a ingerilas espontânea e avidamente sem a necessidade de misturá-las a outros alimentos (Soares et al. 2001).

$O$ diagnóstico da intoxicação por $P$. flavipes em suínos deve ser feito pelo conjunto de informações e sinais clínicos, levando em consideração a época do ano (maio a julho), que coincide com o aparecimento da lagarta nas pastagens e principalmente pelas lesões hepáticas associadas a presença da larva no estômago dos animais. Para diagnóstico diferencial deve-se considerar a intoxicação por Xanthium spp. (carrapicho) (Gava et al. 2005), que em suínos pode ocorrer através de rações, formuladas principalmente com silagem de grão úmido de milho contaminado pelos frutos dessa planta ou pela ingestão dos cotilédones (Stuart et al. 1981, Timm \& Riet-Correa 1997). As lesões histológicas nestes casos são semelhantes, porém o diagnóstico diferencial pode ser realizado pelos dados epidemiológicos. A intoxicação por sementes de Crotalaria spectabilis, pode produzir lesões hepáticas agudas e crônicas em suínos (Torres et al. 1997). No entanto, não há citação de intoxicação espontânea por esta planta no Brasil. Deve se diferenciar, também, da hepatose dietética por deficiência de vitamina $E$ e/ou selênio que ocorre mais frequentemente em suínos jovens e de crescimento rápido (Moreno et al. 2007). Neste caso a necrose hepática é acompanhada por hemorragia grave e as lesões não são uniformes em todos os lóbulos.

\section{REFERÊNCIAS}

Camargo O.R. 1956. As larvas "mata-porco" no Rio Grande do Sul. Bolm Diretoria Prod. Anim. 13:18-25.

Costa Lima A. 1941. Bichos "mata-porcos". Chácaras e Quintais 63:43. Dadswell L.P., Abbott W.D. \& McKenzie R.A. 1984. The occurrence, cost and control of sawfly larval (Lophyrotoma interrupta) poisoning of cattle in Queensland 1972/81. Aust. Vet. J. 62(3):94-97.

Dutra F., Riet-Correa F., Mendez M.C. \& Paiva N. 1997. Poisoning of cattle and sheep in Uruguay by sawfly (Perreyia flavipes) larvae. Vet. Human. Toxicol. 39(5):281-286.

Gava D., Neves D.S., Gava A. \& Cristani J. 2005. Efeitos tóxicos e alterações clínicas, patológicas e hematológicas produzidas por Xanthium spp. em suínos. Anais XII Abraves, Fortaleza, p.153-154.

Jones T.C., Hunt R.D. \& King N.W. 2000. Patologia Veterinária. $6^{\underline{a}}$ ed. Manole, Barueri. 1415p.

McKenzie R.A., Rogers R.J. \& Oelrichs P.B. 1985. Sawfly larvae poisoning of cattle in Queesland. Proc. Australia-USA Poisonous Plants Symposium, Queensland Poisonous Plants Committee, Brisbane, p.524-532.

Moreno A.M., Sobestiansky J. \& Mores N. 2007. Deficiências nutricionais, p.510-524. In: Sobestiansky J. \& Barcellos D. (Eds), Doenças dos Suínos. Cânone Editorial, Goiânia.

Radostits O.M., Gay C.C., Blood D.C. \& Hinchcliff K.W. 2002. Clínica Veterinária: um tratado de doenças dos bovinos, ovinos, suínos, caprinos e eqüinos. 9aa ed. Guanabara Koogan, Rio de Janeiro. 1737p.

Raymundo D.L., Bezerra Jr P.S., Bandarra P.M., Pedroso P.M.O., Oliveira E.C., Pescador C.A. \& Driemeier D. 2008. Spontaneous poisoning by larvae of Perreyia flavipes (Pergidae) in sheep. Pesq. Vet. Bras. 28(1):19-22.

Raymundo D.L., Bezerra Junior P.S., Bandarra P.M., Santos A.S., Sonne L., Pavarini S.P., Côrrea A.M.R., Dias M.M. \& Driemeier D. 2009. Intoxicação espontânea pelas larvas de Perreyia flavipes em bovinos no Estado de Santa Catarina, Brasil. Ciencia Rural. 39(1):163166.

Riet-Correa F., Dutra F., Soares M.P. \& Mendez M.C. 1998. Sawfly (Perreyia flavipes) larval poisoning of cattle, sheep and swine, p.291296. In: Garland T. \& Barr A.C. (Eds), Toxic Plants and Other Natural Toxicants. CAB International Press, New York.

Soares M.P., Riet-Correa F., Smith D.R., Pereira Soares M., Mendez M.C. \& Brandolt A.L. 2001. Experimental intoxication by larvae of Perreyia flavipes Know, 1899 (Hymenoptera: Pergidae) in pigs and some aspects on its biology. Toxicon 39(5):669-678.

Soares M.P., Quevedo P.S. \& Schild A.L. 2008. Intoxicação por larvas de Perreyia flavipes Konow, 1899. Hymenoptera: Pergidae) em bovinos na região sul do Rio Grande do Sul. Pesq. Vet. Bras. 28(3):169173.

Stuart B.P., Cole R.J. \& Gosser H.S. 1981. Cocklebur (Xanthium strumarium var. strumarium) intoxication in swine: Review and redefinition of the toxic principle. Vet. Pathol. 18:368-383.

Torres M.B.A.M., Salles M.W.S., Headley S.A. \& Barros C.S L. 1997. Intoxicação experimental por sementes de Crotalaria spectabilis (Leguminosae) em suínos. Ciência Rural 27(2):307-312.

Timm C.D. \& Riet-Correa F. 1997. Plantas tóxicas para suínos. Ciência Rural 27(3):521-528.

Tramsborg S.M., Jorgensen R.J. \& Brummerstedt E. 1987. Sawfly poisoning in sheep and goats. Vet. Rec. 12:253-255. 